\title{
POLARIZATION OF SOLAR RADIO BURSTS
}

\author{
T. HATANAKA
}

Tokyo Astronomical Observatory, Mitaka, Tokyo, Japan

\section{TIME-SHARING RADIO POLARIMETER}

The polarization observation of solar radio emission at $200 \mathrm{Mc}$./s. was started in December 1954 at the Tokyo Astronomical Observatory with a new type of radio polarimeter [1,2]. With a pair of crossed dipoles at the focus of a Io metre paraboloid of equatorial mounting, it gives almost simultaneously six components of polarization, two circular and four linear, on a time-sharing basis by using electronic switching. Fig. I shows the block diagram of the circuit and the combination of modulators and demodulators. 6AS6 pentodes are used as the time modulators. The duration of each gate pulse is $\mathrm{I} / \mathrm{I} 600 \mathrm{sec}$. and one set of observations is obtained in $\mathrm{I} / 200 \mathrm{sec}$. One of the principal features of the present scheme is to use a common receiving system for all the components in order to avoid inevitable errors due to differences or changes in the gain and in the central frequencies of different receivers. A new type of square-law detector is employed in the present system [3]. It has rapid response time and excellent stability.

Six pieces of information are fed to a six-pen recorder made specially for our purpose. At the same time six Y.E.W. recorders, which are quite similar to Esterline-Angus recorders, are used in order to get a wider latitude in intensity. The overall time-constant is $\mathrm{I} / 2 \mathrm{sec}$. for the former and $\mathrm{I} / 5 \mathrm{sec}$. for the latter. The speed of tape for the former recording is $60 \mathrm{~mm} . / \mathrm{min}$. in active periods. Examples of the observed records are shown in Fig. 2.

\section{SIX GOMPONENTS OF POLARIZATION}

It is known that four independent pieces of information are necessary in order to determine completely the state of polarization. It seems, however, that no such observation has been carried out in radio astronomy. It has been customary to observe two circularly polarized components and at 

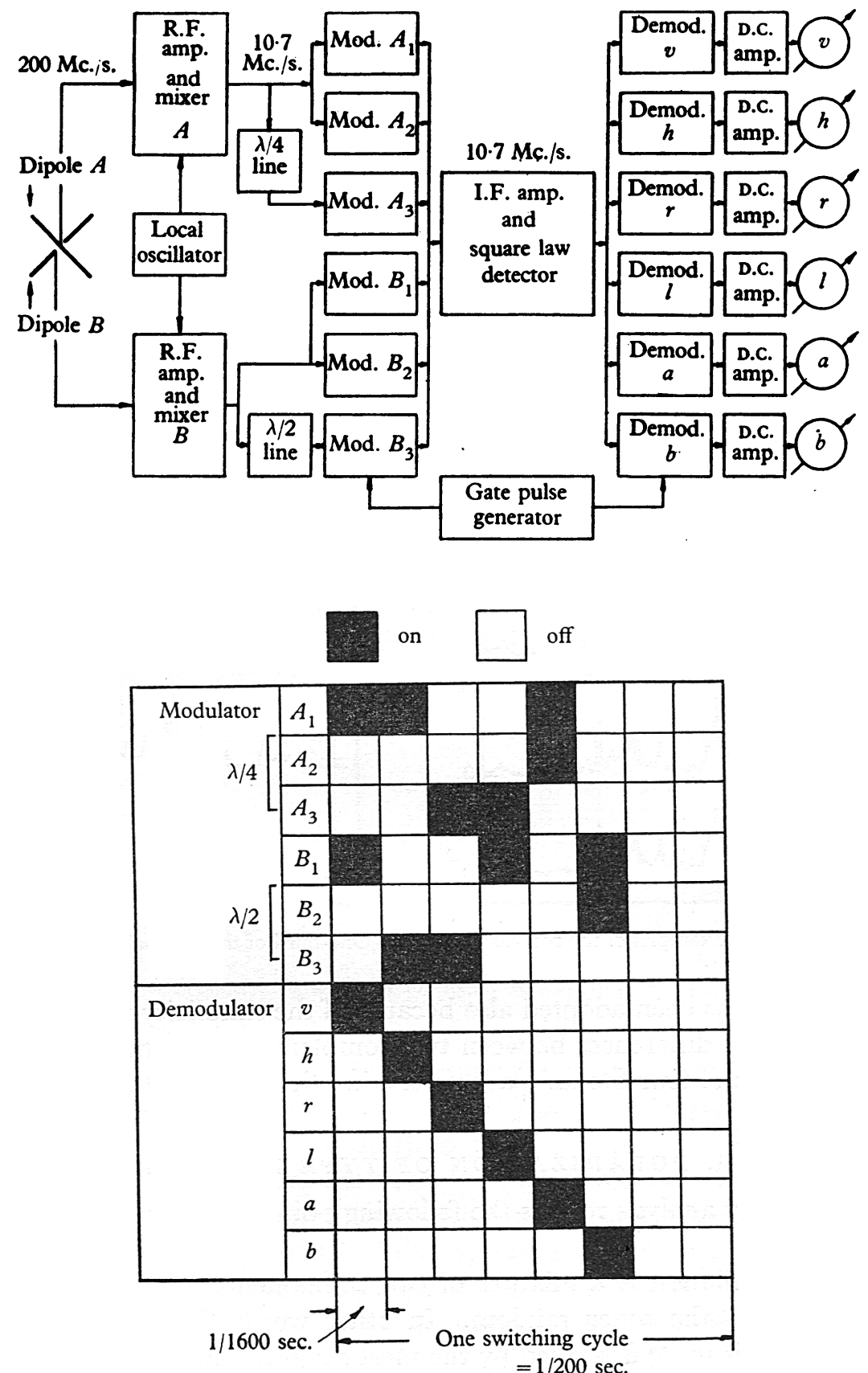

Fig. 1. Block diagram of the circuit and the combination of modulators and demodulators. 
most to add one linearly polarized component [4]. The polarization of solar radio bursts, therefore, has been said to be either random or circular or at most a mixture of circular plus random.

The present system is to observe six components of polarization. This means that our system gives two checks besides necessary information.
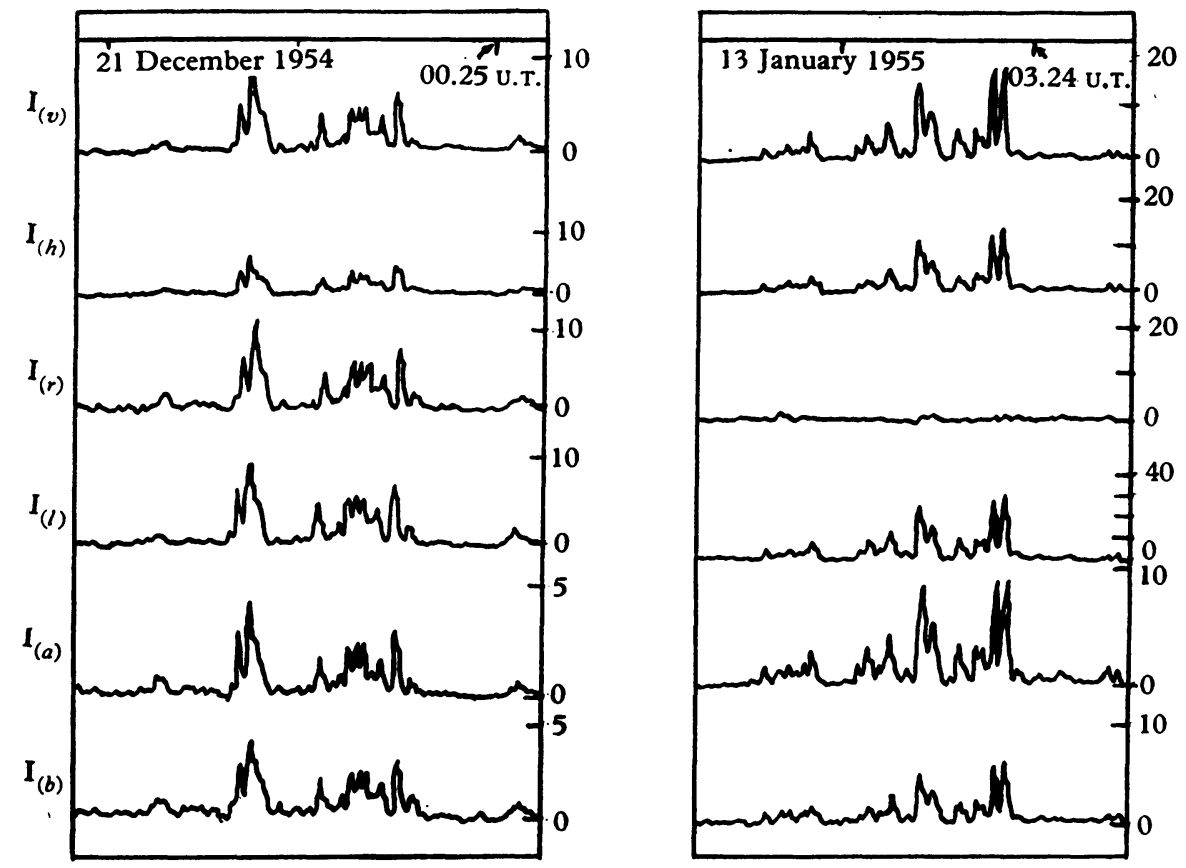

Fig. 2. Examples of the records of bursts. (One-third of the original size.)

This system has been adopted also because of the simplicity in the reduction, since the differences between two complementary components give directly three of four Stokes' parameters, i.e. $Q, U$ and $V[2]$.

\section{POLARIZATION OF TYPE I BURSTS}

A preliminary analysis reveals the following points on the polarization of type I bursts.

(i) The radiation is a mixture of two components: one is elliptically polarized and the other random. In other words the polarization of type $\mathrm{I}$ bursts is to be described by the most general state of polarization.

(ii) The ellipticity varies from nearly $100 \%$, i.e. circular, to $10 \%$, i.e. nearly linear. The variation from day to day is, on the average, similar to 
the ellipticity of a circle put tangentially on the sun's surface at the position of the source and viewed from the earth. The situation is illustrated in Fig. 3. The curves in the figure give the expected ellipticity according to the hypothesis mentioned above. The dotted part means that the source was not active during the period as judged from the interferometer observation at Cornell.
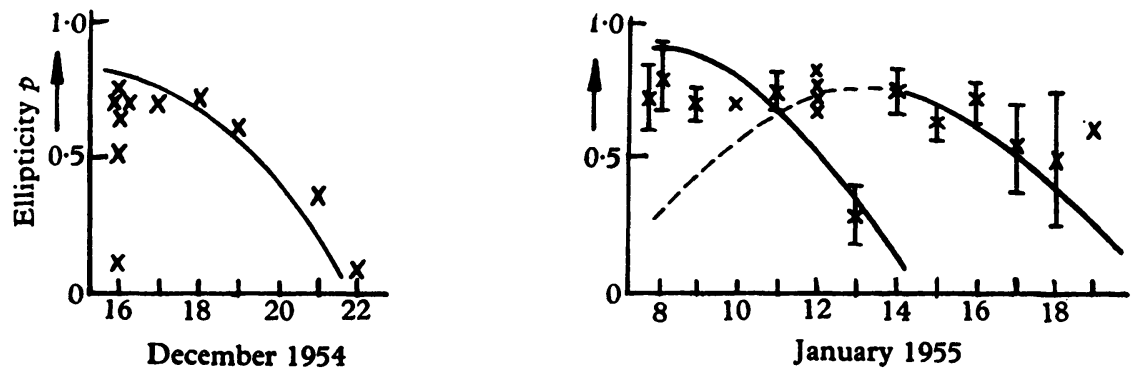

Fig. 3. Variation of the ellipticity with the position of the source.
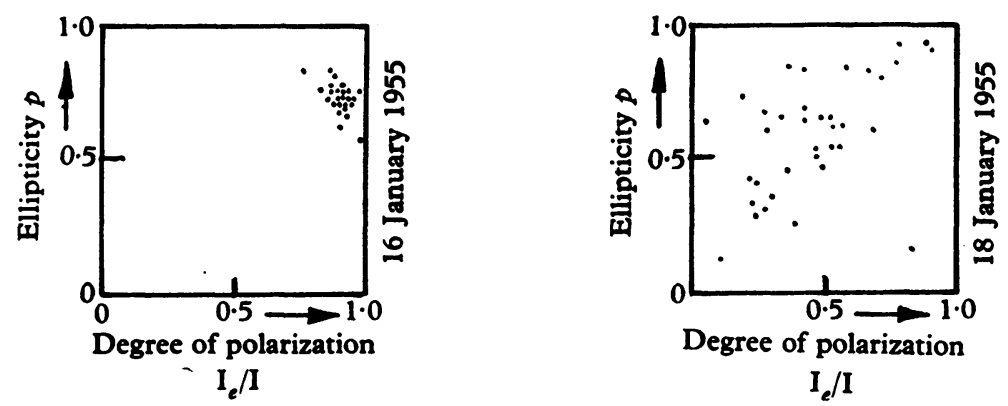

Fig. 4. Correlation between the ellipticity and the degree of polarization.

(iii) The degree of polarization, or the fraction of the elliptically polarized component, is more than $90 \%$ on most days, but sometimes drops below $50 \%$ and even down to $10 \%$.

(iv) The ellipticity and the degree of polarization of bursts are usually the same in one day within the accuracy of the determination, but sometimes they show large scatter. Examples are shown in Fig. 4. It seems that large scatter in such a diagram is observed either on the day the source becomes suddenly active, or when the source is very close to the limb.

(v) The tilt angle of the ellipse is almost constant during the observing time in a day, but is different on different days. 
It is pointed out that the tilt angle of the polarization ellipse is rotated to some extent because of the Faraday effect in the earth's atmosphere. The amount of the rotation is estimated to be several radians at $200 \mathrm{Mc}$./s. for a model ionosphere. It is to be remarked that if the solar radio emission is observed in such a direction that it passes the ionosphere almost transversely to the earth's magnetic field a change in the shape of ellipse besides the rotation of the axis is to be observed. The frequency of $200 \mathrm{Mc}$./s. is, however, too high to observe this 'transverse magnetic field effect'. Such an observation at much lower frequency, say at $50 \mathrm{Mc}$./s., will be of interest [5].

The Faraday effect is also expected to be present in the solar atmosphere. If we adopt a model solar corona and the general solar magnetic field of a dipole type with the polar field of a few gauss the amount of rotation of the polarization ellipse becomes so large that the amount of differential rotation within the receiver band-width becomes conceivable. This will imply a change in the ellipticity and the degree of polarization by passing through the solar corona. This 'finite band-width effect' will be of importance in the theory of the emission and propagation of bursts and also for the structure of the solar atmosphere.

Part of the present research has been carried out during the author's stay at the School of Electrical Engineering, Cornell University.

\section{REFERENCES}

[1] Hatanaka, T., Suzuki, S. and Tsuchiya, A. Proc. Japan Academy, 31, 8I, I955.

[2] Hatanaka, T., Suzuki, S. and Tsuchiya, A. Publ. Astr. Soc. Japan, 7, 1 14, 1955.

[3] Suzuki, S. Publ. Astr. Soc. Japan, 7, 12 1, 1955.

[4] Hatanaka, T. Research Report EE-1 79, Cornell University, 1953.

[5] Hatanaka, T. Research Report EE-257, Cornell University, 1955. Also Hatanaka, T. Publ. Astr. Soc. Japan, 8, 73, 1956.

\section{Discussion}

Wild: Recent records of noise storms at Sydney confirm that at times there is a prolonged period of linear polarization. 